\title{
Surgery versus stereotactic body radiation therapy: Definitive evidence is still elusive
}

\author{
Tara R. Semenkovich, MD, MPHS, and Varun Puri, MD, MSCI
}

\footnotetext{
From the Division of Cardiothoracic Surgery, Department of Surgery, Washington University in St Louis, St Louis, Mo.

Disclosures: Dr Semenkovich was supported by a Barnes-Jewish Hospital Foundation Grant, National Institutes of Health (NIH) Grant Number 2T32HL7776-21, and the Division of Cardiothoracic Surgery at Washington University in St Louis. Dr Puri has nothing to disclose with regard to commercial support.

Received for publication Oct 4, 2018; accepted for publication Oct 4, 2018.

Address for reprints: Tara R. Semenkovich, MD, MPHS, Division of Cardiothoracic Surgery Washington University in St. Louis, 660 S. Euclid Ave, Campus Box 8109, St Louis, MO 63110 (E-mail: semenkovicht@wudosis. wustl.edu).

J Thorac Cardiovasc Surg 2019;157:374-5

$0022-5223 / \$ 36.00$

Copyright (c) 2018 Published by Elsevier Inc. on behalf of The American Association for Thoracic Surgery https://doi.org/10.1016/j.jtcvs.2018.10.012
}

In this issue of the Journal, Cao and colleagues ${ }^{1}$ performed a systematic review and meta-analysis to compare survival outcomes for patients with early-stage lung cancer treated with stereotactic body radiation therapy (SBRT) or surgery in both matched and unmatched cohorts. The authors should be congratulated on their efforts to shed additional light on the ongoing question of SBRT versus surgery for high-risk but operable patients. Their manuscript synthesizes the existing body of literature on the subject using rigorous methodology, and this represents an important contribution to the field. In addition, their thoughtful analyses of both matched and unmatched studies serve as a sensitivity analysis regarding the magnitude of expected benefit from surgery.

Systematic reviews and meta-analyses, although regarded as providing the greatest level of evidence, are summative works and can be influenced by flaws present in the studies used to generate them. Retrospective studies are particularly susceptible to treatment selection bias. Propensity matching can mitigate this bias, but only if all variables that predict treatment selection and affect outcome are included. Here, a minority of studies matched based on pulmonary function tests and functional status, which are critically important variables in determining operative suitability. Comorbidities were largely captured in overall indices, which may not adequately risk-stratify patients. Tumor size and location, which can determine the operative approach and extent of resection required, were also frequently missing. Patient selection is a nuanced process: large national datasets or even institutional databases may fail to capture the major drivers of clinical decisionmaking for individual patients. Consequently, there are likely residual important baseline differences in the surgery and SBRT cohorts within this manuscript, and the reported overall outcomes need to be interpreted in this context. sampling. ${ }^{4}$

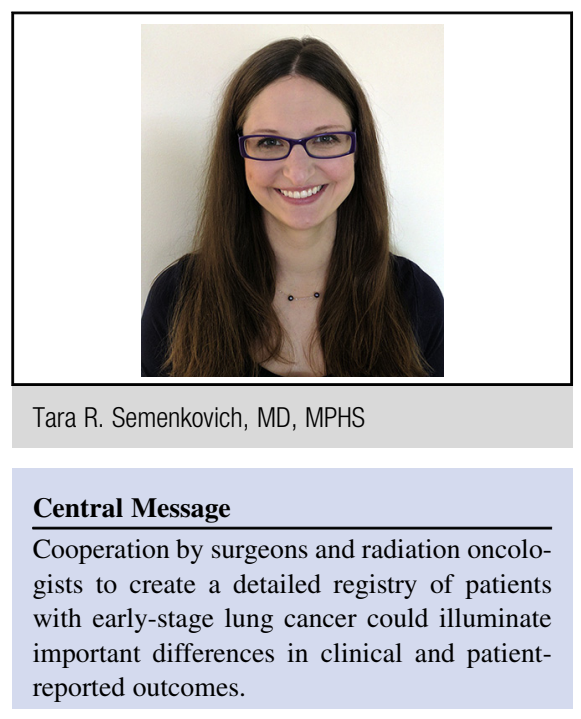

See Article page 362.

At the core of the decision whether to offer surgery or SBRT for an individual patient is an assessment of periprocedural risk: the likely short-term outcomes. The high-risk operative patient population is notoriously difficult to define, and an evidence-based classification or consensus characterization does not exist within the field. Furthermore, an institutional study showed patients who met an American College of Surgery Oncology Group trial definition of high risk had comparable morbidity and mortality with those deemed standard risk. ${ }^{2}$ Sufficiently granular data are lacking to understand exactly how high risk the patients included in this meta-analysis were for both the surgery and SBRT cohorts.

Also factoring heavily into the treatment decision is the predicted risk of mortality due to lung cancer versus competing comorbidities. For patients deemed potentially operable, weighing the potential long-term outcomes necessitates consideration of the likelihood of cancer cure with each treatment option. Cao and colleagues ${ }^{1}$ found significantly improved locoregional control with surgery versus SBRT, confirming previous findings from well-matched institutional cohorts. ${ }^{3}$ Although not assessed in detail in this meta-analysis, these outcomes are likely dependent on performance of a quality operation, which includes negative margins, an anatomic resection when feasible, a minimally invasive operation, and appropriate lymph node 
This work by Cao and colleagues ${ }^{1}$ consolidates the existing knowledge within our field on survival and cancerspecific outcomes for patients with early-stage lung cancer treated with SBRT or surgery. It also, however, clearly exposes significant limitations of the published literature and again demonstrates the need for additional research in this area. Because multiple randomized trials have failed to accrue patients, additional data on the subject are likely to arise through observational studies. Thoracic surgeons should work together with our radiation oncology colleagues moving forward to capture clinical and patientreported outcomes in a large registry. This collaborative method is the most likely to reveal additional relevant details on appropriate patient selection, risk-stratification, and anticipated outcomes, which are currently notably lacking from our literature.

\section{References}

1. Cao C, Wang D, Chung C, Tian D, Rimner A, Huang J, et al. A systematic review and meta-analysis of stereotactic body radiation therapy versus surgery for patients with non-small cell lung cancer. J Thorac Cardiovasc Surg. 2019;157: 362-73.e8

2. Puri V, Crabtree TD, Bell JM, Kreisel D, Krupnick AS, Broderick S, et al. National Cooperative Group Trials of "high-risk" patients with lung cancer: are they truly "high-risk"? Ann Thorac Surg. 2014;97:1678-85.

3. Crabtree TD, Puri V, Robinson C, Bradley J, Broderick S, Patterson GA, et al Analysis of first recurrence and survival in patients with stage I non-small cell lung cancer treated with surgical resection or stereotactic radiation therapy. $J$ Thorac Cardiovasc Surg. 2014;147:1183-91; discussion 1191-2.

4. Hudson J, Semenkovich T, Puri V. Oncologic quality indicators in thoracic surgery. Thorac Surg Clin. 2017;27:227-44. 